\title{
ENGEL-LIKE ELEMENTS IN INFINITE SOLUBLE GROUPS
}

\author{
by ROLF R. A. BRANDL and CHRISTOPHER J. B. BROOKES
}

(Received 9th June 1987)

In [1] the first author considered the following Engel-like condition for a pair of elements $x, y$ of a group $G$.

$$
\text { There exist } r=r(x, y) \geqq 0 \text { and } d=d(x, y) \geqq 1 \text { such that }[x, r y]=\left[x,{ }_{r}+d y\right] .
$$

He studied the situation where (*) is satisfied by all pairs of elements in a soluble group and proved that this is precisely equivalent to the group being locally finite-by-nilpotent, a result analogous to the fact, established by Gruenberg in [3], that a soluble Engel group is locally nilpotent.

Just as in the case of the stronger Engel condition, (*) gives rise for an arbitrary group to two sets of elements: $A(G)$, the set of all $y$ in $G$ such that $(*)$ holds for all $x$ in $G$, so that $A(\mathrm{G})$ contains the set $L(G)$ of all left Engel elements of $G$, and $B(G)$, the set of all $x$ in $G$ such that (*) holds for all $y$ in $G$, so that $B(G)$ contains the set $R(G)$ of all right Engel elements of $G$. Moreover, as in [4], one can show that $x \in B(G)$ implies $x^{-1} \in A(G)$.

The object of this paper is to study some of the properties of $A(G)$ and $B(G)$ for soluble groups $G$. In [3] Gruenberg showed that if $G$ is a soluble group both $L(G)$ and $R(G)$ are subgroups. However it is not hard to see (Example 1) that $A(G)$ is not in general a subgroup, not even for metabelian groups $G$. Not all is lost though; for any group $G$, if we define $A^{*}(G)$ to be the set of all $z$ in $G$ such that (*) holds for all powers $y=z^{i}$ of $z$ and all $x$ in $G$, then we have, as a direct consequence of the proof of Proposition 2 below, the fact that:

If $G$ is a locally soluble group then $A^{*}(G)$ is a subgroup, in fact the unique largest normal locally finite-by-nilpotent subgroup of $G$.

For $B(G)$ the situation is better. As our first main result we prove:

Theorem A. Let $G$ be a locally soluble group. Then $B(G)$ is a characteristic subgroup of $G$ and $B(G / B(G))=1$.

In [2] the second author proved that in a finitely generated soluble group $G$ the set of right Engel elements coincides with the hypercentre $Z_{\infty}(G)$ of $G$. Using the same basic method we shall prove: 
Theorem B. Let $G$ be a finitely generated soluble group. Then $F(G) \leqq B(G)$ and $B(G) / F(G)=Z_{\infty}(G / F(G))$.

Here $F(\mathrm{G})$ denotes the subgroup generated by all finite normal subgroups of $G$.

The behaviour of Engel-like elements is well-illustrated by the class of abelian-bycyclic groups. Let $A$ be a torsion-free abelian normal subgroup of a group $H$ so that there is an element $x$ in $H$ with $H=\langle A, x\rangle$. The following example shows that it is possible for $x$ to lie in $A(G)$ without $x^{-1}$ doing so.

Example 1. Let

$$
H=\left\{\left(\begin{array}{ll}
\alpha & \beta \\
0 & 1
\end{array}\right): \alpha, \beta \in \mathbb{C}, \alpha \neq 0\right\}
$$

and let

$$
x=\left(\begin{array}{cc}
1+i & 0 \\
0 & 1
\end{array}\right)
$$

As $-1+x=(-1+x)^{5}$ we see that $x \in A(H)$. However

$$
-1+x^{-1}=\left(\begin{array}{cc}
(-1-i) / 2 & 0 \\
0 & 0
\end{array}\right)
$$

and $|(-1-i) / 2|<1$. So $\left(-1+x^{-1}\right)^{r}=\left(-1+x^{-1}\right)^{r+d}$ if and only if $d=0$. Hence $x^{-1} \notin A(H)$.

On the other hand making the stronger assertion that $x \in A^{*}(H)$ is enough to ensure that $x$ is actually a left Engel element of $H$ and so $\langle x\rangle \leqq L(H)$. This is immediate from the following basic result about Engel-like elements.

Lemma 1. ([1, Lemma 1]). Let $A$ be a torsion-free abelian group and let $Y$ be a cyclic subgroup of $\operatorname{Aut}(A)$. Assume that for each $a \in A$ and each $y \in Y$ there exist positive integers $r<s$ such that $a^{(-1+y)^{r}}=a^{(-1+y)^{s}}$. If $Y$ is finite then $Y=1$; if $Y$ is infinite then for each $a \in A$ there exists a positive integer $r=r(a)$ with $a^{(-1+y)^{r}}=1$ for all $y \in Y$.

Lemma 1 can also be used as follows to deduce that if $x \in B(H)$ then it is also a left Engel element.

Lemma 2. Let $A$ be a torsion-free abelian normal subgroup of a group $H$ and let $x \in B(H)$. Then for all $a \in A$ there exists a positive integer $r=r(a, x)$ such that $a^{(-1+x) r}=1$. Moreover if $x^{t} \in C_{H}(a)$ for some $t \geqq 1$ then $[a, x]=1$. 
Proof. For $a \in A$ and $i \in \mathbb{Z}$ we have $\left[x,{ }_{k} x^{i} a\right]=\left[x, a,{ }_{k-1} x^{i}\right]$. Hence there exist positive integers $r=r(i, a)<s=s(i, a)$ such that

$$
a^{(-1+x)\left(-1+x^{i}\right)^{r-1}}=a^{(-1+x)\left(-1+x^{i}\right)^{s-1}}
$$

and so

$$
a^{\left(-1+x^{i}\right) r}=a^{\left(-1+x^{i}\right)} .
$$

So Lemma 1 implies $a^{(-1+x) r}=1$ for some $r=r(1, a)$, and $[a, x]=1$ if $x^{t} \in C_{H}(a)$ for some $t \geqq 1$.

Proofs. Our first objective will be to prove the first part of Theorem A which we split off as:

Proposition 1. Let $G$ be a locally (soluble-by-finite) group. Then the set $B(G)$ is a subgroup.

This fact will be deduced from:

Proposition 2. Let $H$ be a locally (soluble-by-finite) group. Then the subgroup generated by finitely many elements of $B(H)$ is finite-by-nilpotent.

To facilitate notation, let $\mathscr{F}$ and $L \mathscr{F}$ respectively denote the classes of finite-bynilpotent and locally (finite-by-nilpotent) groups.

We shall need the following factorisation theorem.

Lemma 3. Let $\Gamma=\left\langle x_{1}, \ldots, x_{n}\right\rangle$ be an $\mathscr{F}$-group. Then $\Gamma=\left\langle y_{1}\right\rangle \cdots\left\langle y_{m}\right\rangle$ where $y_{i} \in\left\{x_{1}, \ldots, x_{n}\right\}$ for all $i$.

Proof. As the torsion subgroup of $\Gamma$ is finite, it lies in a product of finitely many subgroups $\left\langle x_{i}\right\rangle$, so assume that $\Gamma$ is torsion-free nilpotent of class $c$, say. Let $A=\gamma_{c}(\Gamma)$. By induction on $c$ we have $\Gamma / A=\left\langle A y_{1}\right\rangle \cdots\left\langle A y_{m}\right\rangle$ where each $y_{i}$ is some $x_{j}$. Setting $P=\left\langle y_{1}\right\rangle \cdots\left\langle y_{m}\right\rangle$ we thus have $\Gamma=A P$. Now there exist elements $a_{i}=\left[g_{i}, h_{i}\right]$ where $g_{i} \in \gamma_{c-1}(\Gamma), h_{i} \in \Gamma$ and $A=\left\langle a_{1}\right\rangle \cdots\left\langle a_{s}\right\rangle$. For each $r \in \mathbb{Z}$ we have $a_{i}^{r}=\left[g_{i}^{r}, h_{i}\right]$. As $g_{i}^{\prime}$ and $h_{i}$ are contained in $A P$, we have $a_{i}^{r} \in P P P P$ and so $A$ is contained in a product of cyclic subgroups generated by some $x_{i}$.

Lemma 3 is a variation on Proposition 1 of Kropholler [5]. Here though, by restricting to $\mathscr{F}$-groups, it is possible to be more precise about the generators of the cyclic subgroups in the factorisation. This is necessary because in Lemma 4 we are given information only about a particular generating set, and not about all elements, of $\Gamma$.

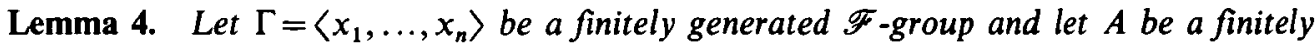


generated $\mathbb{Z} \Gamma$-module. Suppose that a $\mathbb{Z}\left\langle x_{i}\right\rangle$ is a finitely generated $\mathbb{Z}$-module for every $a \in A, 1 \leqq i \leqq n$. Then $A$ is a finitely generated $\mathbb{Z}$-module.

Proof. By Lemma 3, we have $\Gamma=\left\langle y_{1}\right\rangle \cdots\left\langle y_{m}\right\rangle$ where each $y_{j}$ is some $x_{i}$. Let $a \in A$. Then $a \mathbb{Z} \Gamma=a \mathbb{Z}\left\langle y_{1}\right\rangle \cdots\left\langle y_{m}\right\rangle$ and so a simple induction shows that $a \mathbb{Z} \Gamma$ is a finitely generated $\mathbb{Z}$-module. The result follows as $A$ is finitely generated as $\mathbb{Z} \Gamma$-module.

The next result provides a sufficient criterion for a finitely generated soluble group to act nilpotently.

Lemma 5. Let $A$ be a free abelian group of finite rank acted upon by a finitely generated soluble-by-finite group $\Gamma=\left\langle x_{1}, \ldots, x_{n}\right\rangle$. Suppose for each $1 \leqq i \leqq n$ and each $a \in S$ that $\left[a, x_{i}\right]=1$ for some $r=r(a, i) \geqq 1$. Then $[A, t \Gamma]=1$ for some $t \geqq 1$.

Proof. It is enough to show that if $\Gamma$ acts faithfully and rationally irreducibly on $A$ then $\Gamma=1$. By Mal'cev's Theorem (see [7]), $\Gamma$ is abelian-by-finite, so there exists an abelian normal subgroup $\Gamma_{0}$ of $\Gamma$ of finite index $m$. This implies that $\Gamma_{1}=\left\langle x_{1}^{m}, \ldots, x_{n}^{m}\right\rangle^{\Gamma}$ is abelian and can be generated by finitely many elements each of which has the form $y=\left(x_{i}^{m}\right)^{y}$ for some $i$ and $\gamma \in \Gamma$. Moreover for any $a \in A$ there is a positive integer $r$ such that $[a, r y]=1$.

By Clifford's Theorem, $A \otimes \mathbb{Q}$ is a direct sum of irreducible $\mathbb{Q} \Gamma_{1}$-modules. Since $\Gamma_{1}$ is abelian and generated by elements acting unipotently, it acts trivially on such a simple $\mathbb{Q} \Gamma_{1}$-module, so $\Gamma_{1}=1$. Hence $\Gamma$ is finite and Lemma 2 finally implies $\Gamma=1$.

We can now prove Proposition 2. Note that the following argument also proves the statement about $A^{*}(G)$ in the introduction, provided that the definition of $A^{*}(G)$ is appealed to, instead of Lemma 2.

Proof of Proposition 2. Let $H$ be a counterexample and let $x_{1}, \ldots, x_{n} \in B(H)$ be such that $\left\langle x_{1}, \ldots, x_{n}\right\rangle$ is not an $\mathscr{F}$-group. Without loss we may assume $H=\left\langle x_{1}, \ldots, x_{n}\right\rangle$. As finitely generated $\mathscr{F}$-groups are finitely presented, we may assume that every proper quotient of $H$ is an $\mathscr{F}$-group. Let $A$ be a nontrivial abelian normal subgroup of $H$. As $H / A$ is finitely presented, $A$ is a finitely generated $\mathbb{Z}(H / A)$-module. So Lemma 2 and Lemma 4 imply that $A$ is a finitely generated abelian group. By the choice of $H$ the group $A$ is torsion-free and so Lemma 5 implies $A \leqq Z_{t}(H)$ for some $t \geqq 1$. If $T_{1} / A$ denotes the torsion subgroup of $H / A$ then $T_{1} / A$ is finite and the above implies that $T_{1}$ is an $\mathscr{F}$-group. In particular the torsion subgroup $T_{2}$ of $T_{1}$ is finite, so $T_{2}=1$ again by the choice of $H$. This implies that $H$ is nilpotent, a final contradiction.

In order to deduce Proposition 1 from Proposition 2, we need another series of lemmas.

Lemma 6. Let $u$ and $v$ be elements of $a$ group $G$ and let $0 \leqq r \leqq \infty$. Then $\left\langle\left[u,{ }_{k} v\right] \mid 0 \leqq k \leqq r\right\rangle=\left\langle u^{v^{k}} \mid 0 \leqq k \leqq r\right\rangle$. 
Proof. By induction on $k$ one proves that $[u, k v$ is a product of elements of the form $u^{v^{j}}$ and their inverses where $j \leqq k$ and there is exactly one such term with $j=k$. The result follows.

Lemma 7. Let $G$ be a group, let $a, b \in B(G)$ and let $x \in G$. Define

$$
N=\left\langle\left[a,{ }_{k} x\right],\left[a, x^{-1}\right],\left[b,{ }_{k} x\right],\left[b,{ }_{k} x^{-1}\right] \mid k \geqq 0\right\rangle
$$

and set $U=\langle N, x\rangle$. Then $N$ and $U$ are finitely generated and $N$ is a normal subgroup of $U$.

Proof. As $a, b \in B(G)$, only finitely many of the generators of $N$ are distinct. Moreover, Lemma 6 implies $N=\left\langle a^{x^{k}}, b^{x^{k}} \mid k \in \mathbb{Z}\right\rangle$ and hence $N=N^{x}$.

Lemma 8. $U / N^{\prime}$ is an $\mathscr{F}$-group.

Proof. Let $\bar{U}=U / N^{\prime}$. We show that every pair of elements in $\bar{U}$ satisfies (*). As $U / N$ is abelian, it suffices to show that for any $c \in \bar{N}$ and every power $x^{t}$ of $x$ there exist positive integers $r<s$ such that $\left[c, \bar{x}^{t}\right]=\left[c,{ }_{s} \bar{x}^{t}\right]$. As $\bar{N}$ is abelian, it will be enough to prove this for all elements $c$ contained in some system of generators of $\bar{N}$. For example, let $c=\left[\bar{a},{ }_{k} \bar{x}^{e}\right]$ where $e= \pm 1$. As $a \in B(G)$, there exist $r<s$ such that $\left[a,{ }_{r} x^{\dagger}\right]=\left[a,{ }_{s} x^{t}\right]$. Hence we have

$$
\left[c, \bar{x}^{t}\right]=\left[\bar{a},{ }_{k} \bar{x}^{e}, \bar{x}^{t}\right]=\left[\bar{a},{ }_{r}{ }^{t},{ }_{k} \bar{x}^{e}\right]=\left[\bar{a},{ }_{s} \bar{x}^{t},{ }_{k} \bar{x}^{e}\right]=\left[c,{ }_{s} \bar{x}^{t}\right]
$$

as $\bar{U}$ is metabelian. The claim now follows from Lemma 7 and the main result of [1].

Proof of Proposition 1. Let $a, b \in B(G)$ and let $x \in G$. Adopting the notation of Lemma 7, we know that $U / N^{\prime}$ is an $\mathscr{F}$-group. Moreover, Lemma 7 and Proposition 2 imply $N \in \mathscr{F}$. Finally, a result of Hall type due to Lennox [6] implies $U \in \mathscr{F}$ and the claim follows.

Factoring out the hypercentre of a group always yields a group with trivial hypercentre. We now prove that there is a corresponding result for the function $B$.

Proposition 3. Let $H$ be a locally (soluble-by-finite) group. If $H / B(H) \in L F$ then $H \in L \mathscr{F}$.

Proof. Let $K$ be a counterexample and let $L$ be a finitely generated subgroup of $K$ that is not an $\mathscr{F}$-group. As $L \cap B(K) \leqq B(L)$, we infer from the main result of [1] that $L / B(L) \in \mathscr{F}$. So we may assume $K$ is finitely generated. If $\bar{K}=K / N$ is a quotient of $K$ then $\overline{B(K)} \leqq B(\bar{K})$ implies $\bar{K} / B(\bar{K}) \in \mathscr{F}$. Thus, as in the proof of Proposition 2, we may assume that every proper quotient of $K$ is an $\mathscr{F}$-group. Moreover $B(K) \neq 1$. 
Now Proposition 1 implies that $B(K)$ is a subgroup, and so we can choose a nontrivial abelian normal subgroup $N$ of $K$ contained in $B(K)$.

We may choose $N$ to be either torsion or torsion-free, so first assume that $N$ is a torsion group, let $a \in N, a \neq 1$ and let $x \in K$. As $a \in B(K)$, the subgroup $\left\langle\left[a,{ }_{k} x\right] \mid k \geqq 0\right\rangle$ of $N$ is finitely generated and so it is finite. Now Lemma 6 implies that $a$ has only finitely many conjugates of the form $a^{x^{k}}$ and so some nontrivial power of $x$ centralises $a$. Hence [1, Lemma 3] implies that $a$ has only finitely many conjugates in $K$ and so $K$ has a nontrivial finite normal subgroup $F$. As $K / F$ is finite-by-nilpotent, the claim follows.

Now allow $N$ to be torsion-free and let $T / N$ be the torsion subgroup of $K / N$. So $T / N$ is finite and Lemma 1 implies $N \leqq Z(T)$. Hence $T^{\prime}$ is a finite normal subgroup of $K$ and, as above, we see that $T$ is torsion-free abelian. Moreover $K / T$ is torsion-free nilpotent.

We now show that $K$ satisfies an Engel condition. Let $x, y \in K$. Then $b=\left[x,{ }_{k} y\right] \in T$ for some $k \geqq 1$. As $T / N$ is finite, we have $b^{n} \in N$ for some $n \geqq 1$. Now Lemma 1 implies $\left[b^{n}, y\right]=1$ as $b^{n} \in B(K)$. As $T$ is abelian, this yields $[b, y]^{n}=1$ and we get $\left[x,{ }_{k}+l y\right]^{n}=1$. But $T$ is torsion-free and so $[x, k+l y]=1$. This shows that $K$ is nilpotent, a final contradiction.

Proposition 4. Let $G$ be a locally (soluble-by-finite) group. Then $B(G / B(G))=1$.

Proof. Let $R / B(G)=B(G / B(G))$ and let $a \in R$. For $x \in G$ we consider $U=\langle a, x\rangle$ and $\bar{U}=U B(G) / B(G)$. As $\bar{a} \in B(\bar{U})$, we infer that $\bar{U} / B(\bar{U})=\langle\bar{x} B(\bar{U})\rangle$ is cyclic. Hence Proposition 3 shows $\bar{U} \in L \mathscr{F}$ and another application of Proposition 3 proves $U \in L \mathscr{F}$. Hence there exist $r<s$ such that $[a, x]=[a, x]$ and so $a \in B(G)$ as required.

This proves Theorem A. For Theorem B we need some more preparation.

Lemma 9. Let $G$ be a finitely generated group and let $P(G)$ be the join of all normal polycyclic subgroups of $G$. Then $G / P(G)$ contains no nontrivial polycyclic normal subgroups.

Proof. Let $P=P(G)$ and let $x \in G \backslash P$ such that $\left\langle x^{G}\right\rangle P / P$ is polycyclic. Let $G=\left\langle g_{1}, \ldots, g_{n}\right\rangle$ and $\left\langle x^{G}\right\rangle P=\left\langle x_{1}, \ldots, x_{m}\right\rangle P$ with $x_{1}=x$. Consider the subgroup $U=$ $\left\langle x_{1}, \ldots, x_{m},\left[x_{i}, g_{j}^{ \pm 1}\right] \mid 1 \leqq i \leqq m, 1 \leqq j \leqq n\right\rangle$. So $U$ is finitely generated and $U / U \cap P$ is polycyclic. Thus $U \cap P$ is finitely generated as a $U$-group.

Let $V=(U \cap P)^{G}$, so $V$ is finitely generated as a $G$-group and because $V \leqq P$, it is polycyclic. We claim that $W=V\left\langle x_{1}, \ldots, x_{m}\right\rangle$ is a normal subgroup of $G$. In fact, it is enough to establish that $W^{g^{\ddagger 1}} \leqq W$ for each $j$. Since $V$ is normal in $G$, we only need to prove $x_{i}^{g^{\ddagger 1}} \in W$. We have $x_{i}^{g^{\ddagger 1}}=x_{i}\left[x_{i}, g_{j}^{ \pm 1}\right]$. Now $\left[x_{i}, g_{j}^{ \pm 1}\right] \in U \leqq\left\langle x_{1}, \ldots, x_{m}\right\rangle P$ by definition of $U$. Hence we get $\left[x_{i}, g_{j}^{ \pm 1}\right]=h_{1} h_{2}$ for some $h_{1} \in\left\langle x_{1}, \ldots, x_{m}\right\rangle$ and $h_{2} \in P$. Now $h_{2}=h_{1}^{-1}\left[x_{i}, g_{j}^{ \pm 1}\right] \in U$ implies $h_{2} \in U \cap P \leqq V$ and so $x_{i}^{g{ }^{1}} \in x_{i} h_{1} V \subseteq\left\langle x_{1}, \ldots, x_{m}\right\rangle V=W$.

We now prove that $W$ is polycyclic. Indeed, we have

$$
W \cap P=V\left\langle x_{1}, \ldots, x_{m}\right\rangle \cap P=V\left(\left\langle x_{1}, \ldots, x_{m}\right\rangle \cap P\right) \leqq V(U \cap P)=V
$$


by Dedekind's law, and so $W \cap P$ is polycyclic. Moreover, by definition of $W$ we have $W / W \cap P \cong\left\langle x_{1}, \ldots, x_{m}\right\rangle P / P$, so $W / W \cap P$ is polycyclic and hence $W$ is polycyclic.

As $x \in W$ and $W$ is normal in $G$, we have $\left\langle x^{G}\right\rangle \leqq W$. So $\left\langle x^{G}\right\rangle$ is a polycyclic normal subgroup of $G$ and thus $x \in P$.

Proof of Theorem B. Let $C / F(G)=Z_{\infty}(G / F(G))$ and suppose $C \lessgtr B(G)$. Let $D / C$ be a nontrivial abelian normal subgroup of $G / C$ with $D \leqq B(G)$. We are going to construct a certain $\mathbb{Z} G$-module $M$ of the form $M=\left\langle x^{G}\right\rangle C / C$ for some $x \in D \backslash C$. Indeed, if $D / C$ contains an element $x C$ of prime order, then we choose this $x$. Otherwise we choose any element $x \in D \backslash C$. For $a \in M, y \in G$ we have $[a, y]=[a, y)$ for some $r<s$ and Lemma 1 implies that $a$ is annihilated by $f(y)$ where $f$ is some nontrivial integral polynomial. Hence $M$ is a finitely generated constrained $\mathbb{Z} G$-module in the sense of [2] and hence $M$ is a finitely generated abelian group. In particular, $M$ is a polycyclic normal subgroup of $G / C$. Now a threefold application of Lemma 9 shows that $F(G), C$ and finally $\left\langle x^{G}\right\rangle C$ lie in $P$. In particular $\left\langle x^{G}\right\rangle$ is finitely generated and $x \in B(G)$ implies that $\left\langle x^{G}\right\rangle$ is finite-by-nilpotent. Let $T$ be its torsion subgroup. By Lemma 5, applied to the upper central factors of $\left\langle x^{G}\right\rangle / T$ (which are torsion-free), we have $\left[\left\langle x^{G}\right\rangle,{ }_{,} G\right] \leqq T$ for some $t \geqq 1$. But $T \leqq F(G)$ and so $\left\langle x^{G}\right\rangle F(G) / F(G) \leqq C / F(G)$. Thus $x \in C$, a contradiction.

\section{REFERENCES}

1. R. Brande, Infinite soluble groups with Engel cycles: a finiteness condition, Math. Z. 182 (1983), 259-264.

2. C. J. B. Brookes, Engel elements of soluble groups, Bull. London Math. Soc. 18 (1986), 7-10.

3. K. W. Gruenberg, The Engel elements of a soluble group, Illinois J. Math. 3 (1959), 151-168.

4. H. Heineken, Eine Bemerkung über engelsche Elemente, Arch. Math. 11 (1960), 321.

5. P. H. KRopholler, On finitely generated soluble groups with no large wreath product sections, Proc. London Math. Soc. (3) 49 (1984), 155-169.

6. J. C. Lennox, On quasinormal subgroups of certain finitely generated groups, Proc. Edinburgh Math. Soc. 26 (1983), 25-28.

7. D. J. S. Robinson, Finiteness Conditions and Generalized Soluble Groups I (Springer, Berlin 1972).

Mathematisches Institut

Am Hubland 12

D-8700 WURzBURG

West Germany

Corpus Christi College CAMbridge CB2 1RH ENGLAND 